\title{
Directional Coupler Based on a Photonic Quasi-Crystal Fiber with Extended Cores
}

\author{
J. P. da Silva ${ }^{1}$, Einstein G. dos Santos ${ }^{2}$ and Marcos T. B. Segundo ${ }^{3}$ \\ ${ }^{1,2,3}$ Department of Electrical Engineering Federal University of Rio Grande do Norte, Natal, Brazil, \\ patroc@ct.ufrn.br,einsantos@ymail.comandmtbsegundo@gmail.com
}

\begin{abstract}
In this paper, an optical coupler composed by a photonic quasi-crystal fiber (PQCF) with two cores is proposed. The structure is obtained by immersion of a quasi-periodic arrangement of air holes replicated twelve-fold in a conventional fiber optical constituted of pure silica. The cores are generated from defects caused due to absence of two air holes on each identical core and horizontally equidistant from the center of PQCF. The chromatic dispersion in each fiber core is ultra-flat around $38.12 \mathrm{ps} . \mathrm{nm}^{-1} \cdot \mathrm{km}^{-1}$ and to verify the power transfer, the signal was launched on the left core and transferred to the right core in a propagation length of approximately $3.78 \mathrm{~cm}$.
\end{abstract}

Index Terms_- Photonic Quasi-Crystal Fiber; Optical Coupler; Extended Cores.

\section{INTRODUCTION}

The basic geometry of a photonic quasi-crystal fiber (PQCF) is formed by an arrange of quasiperiodic air holes whose depth extends along the length of the fiber. These types of optical fibers, represent a class of structures that exhibit an important feature of do not have translational symmetry, normally found in the traditional photonic crystals (CFs), which are structured materials, where their geometrical functions varies periodically in space [1]. The photonic quasi-crystals (PQC) are characterized by an unusual alignment of atoms forming ordered aperiodic structures, having at least two different symmetrical patterns, which also form a gap-free structure, but they are not repeated regularly [2]. Geometrical reasons reduce the possibilities of symmetries, and after an analysis one can conclude that only 6,8,12 and 24-fold symmetries are possible.

As in photonic crystal fibers (PCF), a PQCF can be defined by the geometric parameters $\Lambda$ (pitch), the center-to-center distance between the air holes, and the air hole diameter $d$. In addition, a PQCF is also characterized by the number of times that the matrix formed by the quasi-periodic arrangement, that is basic matrix, is repeated in the core of pure silica. The PQCF proposed in this work is shown in Figure 1 (a). and the basic matrix is shown in Figure 1 (b). In addition, the introduction of symmetries with an aperiodic array of air holes, in a dielectric matrix with low refractive index produces photonic band gaps, i.e., disallowed bands of frequency in which the light propagation is totally banned [3]. In the limit, for small wavelengths $(\Lambda / \lambda \rightarrow \infty)$, the light is forced to remain only in the region of defects due to the total internal reflection phenomenon. The PQCF presents manufacturing configurations that are similar to the processes used in manufacturing the PCF, that is, they are commonly fabricated using pure silica, doped silica or polymeric materials, and the microstructure of the shell is obtained 
with the inclusion of materials with lower refractive index, commonly with holes filled with air or some other material with refractive index lower than the base material [1], [4].

In addition, an important feature of the structures based on photonic quasi-crystals, lies in the fact that the use of repetitions of the basic cell to mount the structure, can present a high degree of symmetry. Considering the significant band-gaps achieved with these models of structures in the optical range, it has the possibility to fabricate photonic devices that enable a reduction in the coupling losses that are associated with the transition of light in the waveguide interface. This characteristic represents an advantage of the use of PQCF in relation to the PCF [5].

In the next section, the finite element formulation including perfect matched layers $(P M L)$ of cylindrical type are briefly presented, concepts for PQCF project are described in section III, numerical results are shown in section IV, and finally the conclusions of this work are presented.

\section{METHOD OF ANALYSIS}

The finite element method (FEM) is a mathematical tool widely used in telecommunications, specifically in the numerical analysis of propagation characteristics in optical fibers [6]. One can define the FEM as a numerical technique for finding approximate solutions to boundary value problems for partial differential equations (PDE). In this work, the FEM is applied to obtain the $n_{\text {eff }}$ effective refractive index from which it is possible to find the chromatic dispersion $D$, which acts directly on the transmission quality of the waveguide [7]. In addition, the Sellmeier coefficients were used in the vector formulation to examine the chromatic dispersion, the variation of the effective index and the effective area of the fundamental mode at different frequencies.

The Sellmeier coefficients can be applied, without loss of generality, directly to the vector formulation. In essence, these coefficients can be calculated from the Helmholtz differential equation, obtained from Maxwell's equations in differential form:

$$
\nabla \times(\stackrel{=}{k} \nabla \times \vec{H})-k_{0}^{2} \vec{H}=0
$$

where $\overline{\bar{k}}=1 /(\overline{\bar{\varepsilon}} \overline{\bar{L}})$, in which $\stackrel{\bar{\varepsilon}}{\bar{\varepsilon}}$ represents the permittivity tensor or dielectric tensor, and $\overline{\bar{L}}$ corresponds to $a$ tensor that relates the parameters of the cylindrical PML [8]. After some algebraic manipulation and assuming that in both medias the fields vary relatively slowly along the propagation direction z [6], the equation (1) can be rewritten as:

$$
[A]\{\phi\}=n_{e f f}^{2}[B]\{\phi\}
$$

where $[\mathrm{A}]$ e $[\mathrm{B}]$ are sparse and complex matrices. Equation (2) is efficiently solved by subspaces method. Here, the FEM is applied together with the vectorial beam propagation method (VBPM) [6] and perfectly matched layer in cylindrical coordinates. 


\section{PQCF DESIGN}

The proposed structure is composed of pure silica and is characterized by a quasi-periodic array with microscopic air holes forming an arrangement with 12-fold symmetry, Figure 1 (a). The air holes are separated by $\Lambda$ and have equal diameters $d$, except for the central hole, which has a varying diameter $d_{c}$. The structure consists of two identical cores horizontally equidistant from the central air hole. The cores are distributed in order to permit maximum energy transfer between them. Figure 1 (b) shows the used quasi-periodic array to design the geometry with repetition of 12-fold symmetry and the hexagonal structure containing the adjusted diameter of the central air hole is shown in Figure 1 (c). It is important emphasize that the $\Lambda$ is usually constant and presents a significant effect on the chromatic dispersion curve localization [8].

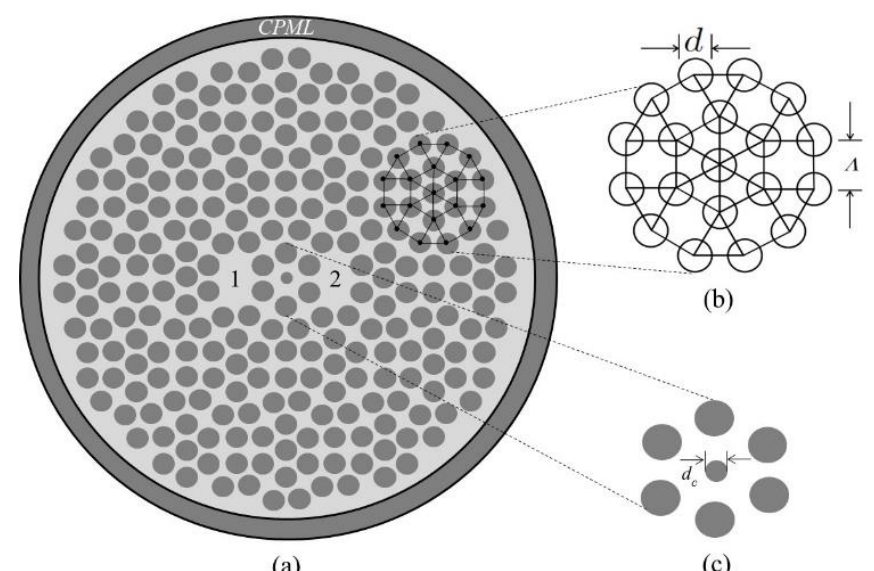

(a)

(c)

Fig. 1 (a) Cross section of a PQCF with 12-fold symmetry and extended and identical cores 1 e 2, (b) Basic matrix of the quasi-periodic array and (c) Central air hole detail.

In the design of this new fiber, the extended cores are obtained by introducing a defect caused by removing two neighboring air holes, located near the central air hole of the quasi-crystalline mesh, which forms the pattern structure of repetitions. In fact, removing the air holes does not affect the symmetry of repetitions along the length of the fiber, since these defects are located at equidistant and symmetrical points in relation to the horizontal line center of the PQCF transversal section. Another point remains in the fact that the photonic quasi-crystals can have their symmetry and geometry predefined, which allowed the development of this design [9]. It is important to emphasize that the crystalline structure was obtained by intercalating air holes, which were immersed in pure silica with a corresponding wavelength of $1.55 \mu \mathrm{m}$.

\section{RESULTS}

In this work, a study was first performed considering the structure composed by just core 1 and then the core 2 . In both cases, the PQCF is formed with 12-fold symmetry and values of $d=1.2 \mu \mathrm{m}, d / \Lambda=$ 0.5 e $\lambda=1.55 \mu \mathrm{m}$. Here, the objective was to verify the effective area of the structure and the chromatic dispersion in each of the cores separately. 
The effective modal area refers to the area of the fiber where optical power is effectively transmitted. To obtain this area in the analyzed PQCF, the energy beam was launched into the left core (core 1), assuming the right core without defects, next the signal was launch into the core 2, considering the left core without defects, the same procedure was adopted to obtain the chromatic dispersion in the cores 1 and 2. Figure 2 (a) shows a comparison between the effective modal area of the left extend core (where the core is obtained due defect caused by removing two air hole) and central core. To obtain the effective area in the central core, the defect was introduced due removing one air hole only in the hexagon of the structure center. In addition to the effective modal area, all simulation were obtained using the expression $A_{\text {eff }}=\left(\iint|E|^{2} d x d y\right)^{2}\left(\iint|E|^{4} d x d y\right)$ [7], where $E$ is the transverse electric field and the material which constitutes the PQCF is pure silica with refractive index of 1.44. To obtain the $A_{\text {eff }}$ and the chromatic dispersion, it was consider $d_{\mathrm{c}}=d$ and the computational window for all simulations was divided into 18,368 linear elements. The effective area for the PQCF in analysis is proportional to the diameter of the modal field. Thus, the PQCF with extended cores have a larger effective area when compared to the single core PQCF in the center of the structure. This way, we can conclude that the PQCF with extended core can transmit a greater amount of power.

To obtain the chromatic dispersion as a function of the wavelength was used the expression $D=-$ $(\lambda / c)\left(d^{2} n_{\text {eff }} / d \lambda^{2}\right)$, where $c$ represents the speed of light in free space. The chromatic dispersion obtained is ultra-flat and varies between 36.75 e $38.125 \mathrm{ps} /(\mathrm{km} . \mathrm{nm})$ for a range of wavelengths from $1.2 \mu \mathrm{m}$ to $1.74 \mu \mathrm{m}$ as shown in Fig. 2 (b).

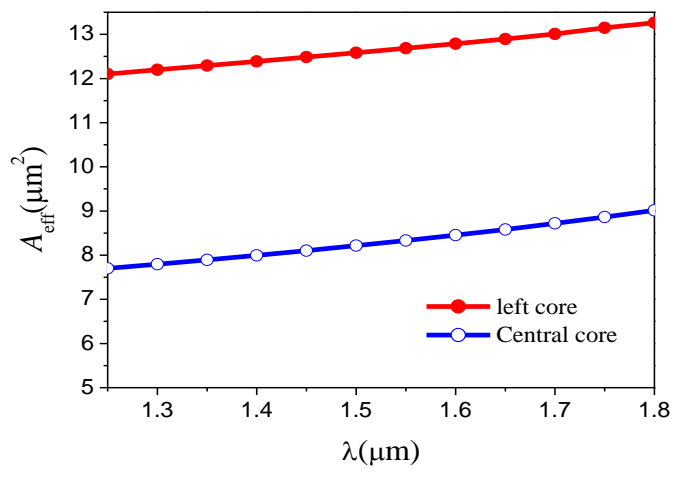

(a)

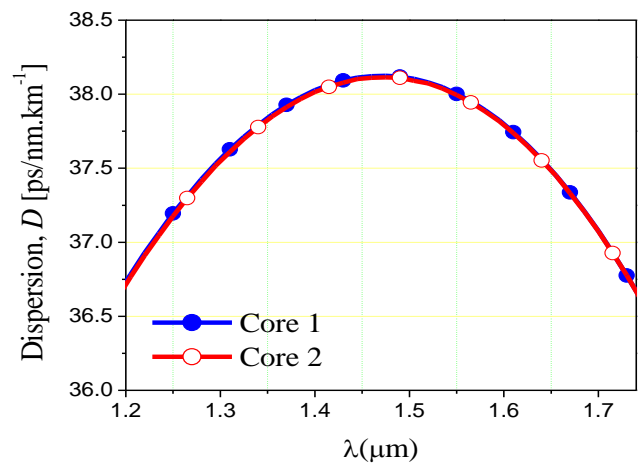

(b)

Fig. 2 (a) Effective Area $\left(A_{\text {eff }}\right)$ of the left and central cores in function of wavelengths from $\lambda=1.25 \mu \mathrm{m}$ to $\lambda=1.8 \mu \mathrm{m}$ and (b) Chromatic dispersion for the structure showed in Fig. 1, considering the analysis for the cores 1 e 2 separately with $d_{\mathrm{c}}=d=$ $1.2 \mu \mathrm{m}$.

In the Figure 2 (b) we can see that the structure presents a flat and positive dispersion to a range of wavelengths for layers $\mathrm{E}+\mathrm{S}+\mathrm{C}+\mathrm{G}+\mathrm{U}$ approximately $37.5 \mathrm{ps} \cdot \mathrm{km}^{-1} \cdot \mathrm{nm}^{-1}$ for the cores 1 and 2 individually. A possible application to the coupling structures analyzed in this paper, could be its use for the power transmission with chromatic dispersion adjustment for the wavelength range considered 
and results obtained in this analysis.

For the second application, we considered the structure shown in Figure 1 (a) with two cores to perform the analysis of the coupling characteristics at the wavelength $\lambda=1.55 \mu \mathrm{m}$. The energy transfer can be controlled by varying the diameter of the air hole $d_{\mathrm{c}}$ introduced into the hexagon located in the center of PQCF. Here, the fiber was excited with the signal launched into the core 1 corresponding to the mode $E_{11}^{x}$, for which the effective refractive index $\left(n_{\text {eff }}\right)$ obtained through modal analysis [10].

The coupling distance obtained from VBPM is in accordance with the distance obtained using the modal analysis through the relation $L_{B}=\pi /\left(\beta_{\text {eff } 1}-\beta_{\text {eff } 2}\right)$ where $L_{\mathrm{B}}$ is the beat length, and $\beta_{\text {eff1 }}$ and $\beta_{\text {eff2 }}$ correspond to the propagation constant of the super-symmetric and anti-symmetric mode of the lowest order. Figure 3 shows the power transfer between the cores 1 and 2 of PQCF along the direction of propagation. The red curve with empty squares corresponds to the power variation in the core 1 and the red curve with filled squares represents the power variation in the core 2 , considering $d_{\mathrm{c}}=0.684$ $\mu \mathrm{m}$. The blue curve with empty circles corresponds to the power variation in the core 1 , the blue curve with filled circles represents the power variation in the core 2 , considering $d_{\mathrm{c}}=0.3 \mu \mathrm{m}$. The black curve with empty triangles corresponds to the power variation in the core 1 and the black curve with filled triangles corresponds to the power variation in the core 2 , considering $d_{\mathrm{c}}=0 \mu \mathrm{m}$.

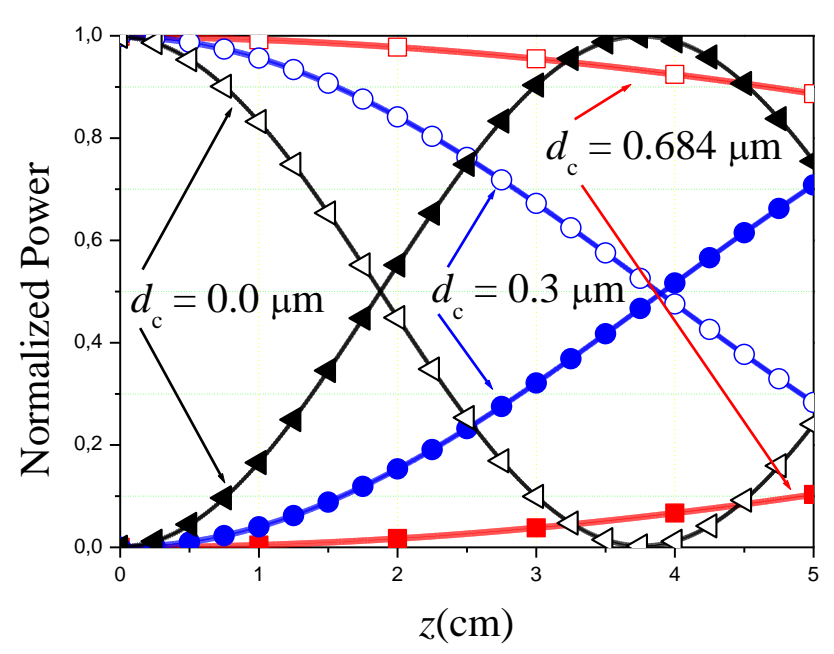

Fig. 3. Distance of maximum power transfer for the PQCF with two cores considering $d_{C}=0.684 \mu \mathrm{m}, d_{C}=0.3 \mu \mathrm{m}$ e $d_{C}=0$ $\mu \mathrm{m}$ para $\lambda_{1}=1.55$ e $d / \Lambda=0.5$.

From the results, it is observed that, for the first diameter $d_{c}=0.648 \mu \mathrm{m}$, the energy launched into the core 1 is totally transferred to the core 2 in the propagation distance of $L_{\mathrm{c}}=23.46 \mathrm{~cm}$. For $d_{\mathrm{c}}=$ $0.300 \mu \mathrm{m}$, the energy launched in core 1 is totally transferred to the core 2 in the propagation distance of $L_{\mathrm{c}}=7.6 \mathrm{~cm}$ and when we consider the central hexagon of PQCF with $d_{\mathrm{c}}=0 \mu \mathrm{m}$, the energy launched into the core 1 is totally transferred to the core 2 in a propagation distance $L_{\mathrm{c}}=3.78 \mathrm{~cm}$.

It is important to note that the absence of defect in the central air hole, where $d_{c}=0 \mu \mathrm{m}$, does not Brazilian Microwave and Optoelectronics Society-SBMO received 26 Oct 2017; for review 26 Oct 2017; accepted 05 Apr 2018 
characterize the inclusion of a third core in fiber, because no energy is stored in the central hexagon during the transfer of energy between the cores 1 and 2. However, when a defect in the core of the central hexagon is caused, there is a high sensitivity in the power transfer between the cores, that is, small variations in the diameter $d_{\mathrm{c}}$ provoke large variations in the coupling distance. Finally, Figure 4 shows the electric field distribution from the simulation with VBPM for propagation distance $\mathrm{z}=0$ and $L_{\mathrm{c}}$ for $\lambda=1.55 \mu \mathrm{m}$.

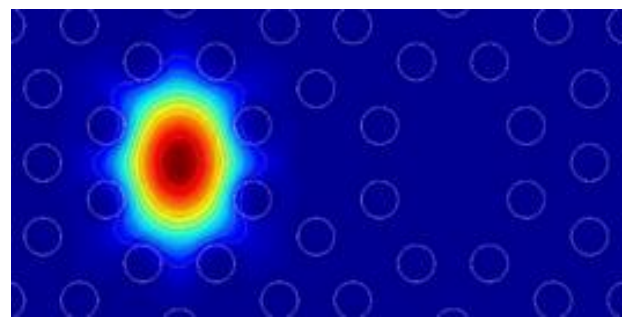

(a)



(b)

Fig. 4. Electric field distribution in (a) $z=0 \mathrm{e}$ (b) $z=L_{\mathrm{c}}$.

In addition, as an application using structures based in photonics quasi-crystals, recently, optical beam couplers were developed from networks photonics quasi-crystals with twelve-folds symmetry. In this case, the device was constructed by random stampfli inflation, etched in silicon oxynitride slab waveguide deposited on silicon dioxide [11]. On the other hand, making a parallel between the structure analyzed in this paper and the couplers based on PQCF, we can consider the advantage of PQCF to facilitate the use of the extended cores due to the arrangement of air holes and its twelvefold symmetry, which does not occur in PCF due to the periodicity of the air holes hexagonal rings distribution.

\section{CONCLUSIONS}

In this work, a new design of an optical coupler formed by a PQCF with two extended cores was proposed. The values of the structural parameters were obtained with the aim of improving the performance of the PQCF optical coupler. The modal analysis of PQCF, considering the cores isolated, was performed in order to obtain a chromatic dispersion in each of the cores of the fiber as being ultra-flat around of $38.12 \mathrm{ps} . \mathrm{nm}^{-1} \cdot \mathrm{km}^{-1}$. In the C-band, that corresponds to the optical wavelength range from $1530 \mathrm{~nm}$ to $1565 \mathrm{~nm}$, the slope of the dispersion curve was around 0.0015 ps.nm ${ }^{-1} \cdot \mathrm{km}^{-2}$. The results obtained show that all the energy launched into the core 1 was transferred to the core 2 of the PQCF in a propagation distance of $3.78 \mathrm{~cm}$ for $d_{\mathrm{c}}=0 \mu \mathrm{m}$. The sensitivity analysis of this fiber relative to the diameter of the central air hole was shown in Figure 3. The variation of these parameters was introduced under the assumptions that the others remain constant. For this analysis, it is clear that for an increase of $5.0 \% d_{\mathrm{c}}$ results in an increase of approximately $10 \%$ in the coupling distance. 


\section{ACKNOWLEDGMENT}

The authors would like to thank the structural support of the UFRN and UFERSA and financial supports of the CAPES and CNPq.

\section{REFERENCES}

[1] S. Kim, C. S. Kee and J. Lee, "Novel Optical Properties of Six-Fold Symmetric Photonic Quasicrystal Fibers", Opt. Express, v. 15, pp. 13221-13226, 2007.

[2] S. Kim and C. S. Kee, "Dispersion Properties of Dual-Core Photonic Quasicrystal Fibers", Optics Express, V. 15, 15885-15890, 2009.

[3] L. Yu-He, F. Wan-De and S. Qiu-Qin, "A novel Photonic Quasicrystal Fiber with Broadband Large Negative Dispersion", Chine Physics Letters, V. 27, 114211-1-4, 2010.

[4] M.J. Gander, R. McBride, J.D.C. Jones, D. Mogilevtsev, T.A. Birks, J.C. Knight, and P.St.J. Russell, "Experimental measurement of group velocity dispersion in photonic crystal fibre,” Electronic Letters, V. 35, 63-64, 1999.

[5] Robert C. Gauthier and Alexei Ivanov, "Production of quasi-crystal template patterns using a dual beam multiple exposure technique", Optical Express, V. 12, 990-1003, 2004.

[6] J. P. da Silva, H. E. Hernández-Figueroa and A. M. F. Frasson, "Vectorial finite-element BPM analysis for transverse anisotropic media”, J. Lightwave Technology, V. 21, 567-576, 2003.

[7] K. Saitoh, N. Florous, and M. Koshiba, "Ultra-flattened chromatic dispersion controllability using a defected-core photonic crystal fiber with low confinement losses," Optics Express V. 13, 8365-8371 (2005).

[8] Xin Cheng, Ming-Jun Li, J. Koh, A. Artuso and D. A. Nolan, "Effects of bending on the performance of hole-assisted single polarization fibers" Optics Express, V. 15, 10619-10636, 2007.

[9] Weicheng Cai. Exian Liu, Bo Feng, H. Liu, Z. Wang, W. Xial T. Liang, S. Wang, J. Liu and Jianjun Liu, “ Dispersion proparties of a photonic quasi-crystal fiber with double cladding air holes”, Elsevier Optik, V. 127, 4438-4442, 2016.

[10] J. P. da Silva and E. R. M. Dantas, “Modal anlysis of a photonic quasi-crystal fiber of silica doped with germanium”, International Microwave \& Optoelectonics Conference (IMOC), 2013 SBMO/IEEE MTT-S, 1-5, 2013.

[11] Jingxing Shi, Michael E. Pollard, Cesar A. Angeles, Ruiqi Chen, J. C. Gates and M. D. B. Charlton, "Photonic crystal and quasi-crystals providing simultaneous light coupling and beam splitting within a low refractive-index slab waveguide”, Scientific Reports, V. 7, 1-10, 2017. 